\title{
On a question concerning D4-modules
}

\author{
S. Das
}

Department of Mathematics, KPR Institute of Engineering and Technology, Coimbatore-641407, India

For citation: Das S. On a question concerning D4-modules. Vestnik of Saint Petersburg University. Mathematics. Mechanics. Astronomy, 2021, vol. 8(66), issue 3, pp. 467-474.

https://doi.org/10.21638/spbu01.2021.308

An $R$-module $M$ is called a D4-module if 'whenever $M_{1}$ and $M_{2}$ are direct summands of $M$ with $M_{1}+M_{2}=M$ and $M_{1} \cong M_{2}$, then $M_{1} \cap M_{2}$ is a direct summand of $M^{\prime}$. Let $M=\oplus_{i \in I} M_{i}$ be a direct sum of submodules $M_{i}$ with $\operatorname{Hom}\left(M_{i}, M_{j}\right)=0$ for distinct $i, j \in I$. We show that $M$ is a $D 4$-module if and only if for each $i \in I$ the module $M_{i}$ is a D4-module. This settles an open question concerning direct sums of D4-modules. Our approach is independent of the solution obtained by D'Este, Keskin Tütüncü and Tribak recently.

Keywords: SIP-modules, D4-modules.

1. Introduction. By a ring we mean an associative ring with an identity element; modules are unitary.

A module $M$ is said to be a SIP-module (SSP-module) if the intersection (respectively, the sum) of two direct summands of $M$ is a direct summand of $M$. Kaplansky observed that over a commutative principal ideal domain every free module is a SIPmodule (see [1, Exercise 51(a), p. 49].) SIP-modules and SSP-modules have been extensively studied (see, for example, [2-4] and [5]).

For $1 \leq i \leq 4$, a module $M$ is called a $D i$-module if it satisfies the condition $D i$ noted below.

$D 1$. For every submodule $A$ of $M$, there is a decomposition $M=M_{1} \oplus M_{2}$ such that $M_{1} \leq A$ and $A \cap M_{2}$ is small in $M_{2}$.

$D 2$. If $A \leq M$ such that $M / A$ is isomorphic to a direct summand of $M$, then $A$ is a direct summand of $M$.

D3. If $M_{1}$ and $M_{2}$ are direct summands of $M$ with $M_{1}+M_{2}=M$, then $M_{1} \cap M_{2}$ is a direct summand of $M$.

D4. If $M_{1}$ and $M_{2}$ are direct summands of $M$ with $M_{1}+M_{2}=M$ and $M_{1} \cong M_{2}$, then $M_{1} \cap M_{2}$ is a direct summand of $M$.

(For a detailed background of these notions, we refer to [6, Chapter 4] and to [7].)

A module $M$ is also called a lifting module if it satisfies condition $D 1$ (see [8] for detailed information regarding these modules). We recall the characterization "the ring $R$ is semiperfect if and only if $R$ is lifting as a right (or left) $R$-module" (see [9, Theorem 1.2.13]). Now let $R$ be a commutative domain with zero Jacobson radical which is not a field, and hence is not semiperfect. Then, by the above results, ${ }_{R} R$ is a projective module which is not a $D 1$-module. We have, however, projective $\Longrightarrow$ quasi-projective $\Longrightarrow$

(C) St. Petersburg State University, 2021 
D2-module $\Longrightarrow D 3$-module $\Longrightarrow D 4$-module (see [6, Proposition 4.38 and Lemma 4.6]). Note that for all proper subgroups $N$ of the (indecomposable) Prüfer $p$-group $M=Z_{p^{\infty}}$, the group $M / N$ is isomorphic to $M$. Hence it is $D 3$ (as a $\mathbb{Z}$-module) but not $D 2$. In fact, there are rings over which every cyclic module is $D 3$ but not all cyclic modules are $D 2$ (see [10, Example 6.4]).

There is no known example of a module which is D4 but not D3 [11] (see also [12, p. 2]).

Let $A$ and $B$ be right $R$-modules. A homomorphism $f \in \operatorname{Hom}_{R}(A, B)$ is said to be (von Neumann) regular (briefly, regular) if for some homomorphism $g \in \operatorname{Hom}_{R}(B, A)$, we have the relation $f=f g f$. It is well-known that a homomorphism $f \in \operatorname{Hom}_{R}(A, B)$ is regular if and only if $\operatorname{Ker}(f)$ is a direct summand in $A$ and $\operatorname{Im}(f)$ is a direct summand in $B$.

Recall that a module $M$ is called a Rickart module if the kernel of any endomorphism $f \in \operatorname{End}_{R}(M)$ is a direct summand in $M$. It follows from [13, Proposition 2.16] that every Rickart module is a SIP-module. A module $M$ is called a dual Rickart module if the image of any endomorphism $f \in \operatorname{End}_{R}(M)$ is a direct summand in $M$. It follows from [14, Proposition 2.11] that every dual Rickart module is a SSP-module.

2. Results. We begin with the recall of some results from [15].

Lemma 1 [15, Lemma 2.1]. Let $M$ be a right $R$-module, $f, g \in \operatorname{End}_{R}(M)$ be regular homomorphisms, and let

$$
M=\operatorname{Ker}(f) \oplus A=\operatorname{Im}(f) \oplus B, M=\operatorname{Ker}(g) \oplus A^{\prime}=\operatorname{Im}(g) \oplus B^{\prime} .
$$

Then the following assertions hold:

(a) $\operatorname{Im}(f g)=f(A \cap(\operatorname{Im}(g)+\operatorname{Ker}(f)))$;

(b) $\operatorname{Ker}(f g)=\left(\left.g\right|_{A^{\prime}}\right)^{-1}(\operatorname{Im}(g) \cap \operatorname{Ker}(f))+\operatorname{Ker}(g)$.

Lemma 2 [15, Lemma 2.2]. Let $M$ be a right $R$-module, $\pi$ be the projection onto the first direct summand with respect to the decomposition $M=A_{1} \oplus A_{2}$, and let $\pi^{\prime}$ be the projection onto the first direct summand with respect to the decomposition $M=B_{1} \oplus B_{2}$. Then the following assertions hold:

(a) $\operatorname{Im}\left(\pi^{\prime} \pi\right)=\left(A_{1}+B_{2}\right) \cap B_{1}$;

(b) $\operatorname{Ker}\left(\pi^{\prime} \pi\right)=\left(A_{1} \cap B_{2}\right)+A_{2}$.

Proposition 1 [15, Theorem 2.3]. For a right $R$-module $M$, the following conditions are equivalent.

1. $M$ is a SSP-module.

2. For any two regular homomorphisms $f, g \in \operatorname{End}_{R}(M)$, the module $\operatorname{Im}(f g)$ is a direct summand of the module $M$.

Proposition 2 [15, Theorem 2.4]. For a right $R$-module $M$, the following conditions are equivalent.

1. $M$ is a SIP-module. 
2. For any two regular homomorphisms $f, g \in \operatorname{End}_{R}(M)$, the module $\operatorname{Ker}(f g)$ is a direct summand of the module $M$.

Next we note examples of finite abelian groups which are not $D 4$.

Example. Consider $M=\mathbb{Z} / 2 \mathbb{Z} \oplus \mathbb{Z} / 4 \mathbb{Z}$ as a $\mathbb{Z}$-module. Then $A=(\overline{1}, \overline{3}) \mathbb{Z}$ and $B=(\overline{0}, \overline{3}) \mathbb{Z}$ are isomorphic direct summands of $M$. However, $A \cap B$ is not a direct summand of $M$. In fact, for any prime $p$, consider $M=\mathbb{Z} / p^{m} \mathbb{Z} \oplus \mathbb{Z} / p^{n} \mathbb{Z}$ with $n>m$ as a $\mathbb{Z}$-module, then $M$ is not a $D 4$-module, since there is an epimorphism $\mathbb{Z} / p^{n} \mathbb{Z} \longrightarrow \mathbb{Z} / p^{m} \mathbb{Z}$ whose kernel is not a direct summand of $\mathbb{Z} / p^{n} \mathbb{Z}$.

The following theorem is an analogue of [15, Theorem 3.3].

Theorem 1. For a right $R$-module $M$, consider the following statements.

1. $M$ is a D3-module.

2. For any two regular endomorphisms $f, g \in \operatorname{End}_{R}(M)$, if $\operatorname{Im}(f g)$ is a direct summand of the module $M$, then the module $\operatorname{Ker}(f g)$ is a direct summand of the module $M$.

3. For any two regular endomorphisms $f, g \in \operatorname{End}_{R}(M)$ satisfying the following:

(i) $\operatorname{Im}(f g)$ is a direct summand of the module $M$,

(ii) $\operatorname{Ker}(f) \cong \operatorname{Im}(g)$,

then the module $\operatorname{Ker}(f g)$ is a direct summand of the module $M$.

4. $M$ is a D4-module.

5. For any two regular endomorphisms $f, g \in \operatorname{End}_{R}(M)$ satisfying the following:

(i) $\operatorname{Im}(f g)$ is a direct summand of the module $M$,

(ii) $N+\operatorname{Ker}(f) \cong \operatorname{Im}(g)$ for any direct summand $N$ of $M$ such that $N \cap \operatorname{Ker}(f)=0$, then the module $\operatorname{Ker}(f g)$ is a direct summand of the module $M$.

Then $(1) \Leftrightarrow(2) \Rightarrow(3) \Rightarrow(4) \Rightarrow(5)$.

Proof. (1) $\Leftrightarrow(2)$ follows from [15, Theorem 3.3].

$(2) \Rightarrow(3)$ is clear.

$(3) \Rightarrow(4)$. Let $M=A \oplus A^{\prime}=B \oplus B^{\prime}$, where $A+B=M$ and $A \cong B$. Consider the natural projections $\pi_{1}: A \oplus A^{\prime} \longrightarrow A$ and $\pi_{2}: B \oplus B^{\prime} \longrightarrow B^{\prime}$. Then by Lemma 2(a), $\operatorname{Im}\left(\pi_{2} \pi_{1}\right)=B^{\prime}$ is a direct summand of $M$. Therefore by assumption and Lemma 2(b), $\operatorname{Ker}\left(\pi_{2} \pi_{1}\right)=(A \cap B) \oplus A^{\prime}$ is a direct summand of $M$. This shows that $A \cap B$ is a direct summand of $M$, as required.

$(4) \Rightarrow(5)$. Let

$$
M=\operatorname{Ker}(f) \oplus A=\operatorname{Im}(f) \oplus B=\operatorname{Ker}(g) \oplus A^{\prime}=\operatorname{Im}(g) \oplus B^{\prime} .
$$

By Lemma $1\left(\right.$ a), since $\left.f\right|_{A}$ is an isomorphism $(\operatorname{Im}(g)+\operatorname{Ker}(f)) \cap A$ is a direct summand of $M$. Therefore, $A=N \oplus(\operatorname{Im}(g)+\operatorname{Ker}(f)) \cap A$, for some $N \leq A$. Since $(N+\operatorname{Ker}(f))+$ $\operatorname{Im}(g)=M, N+\operatorname{Ker}(f) \cong \operatorname{Im}(g)$ and $M$ is a $D 4$-module, we have $(N+\operatorname{Ker}(f)) \cap$ 
$\operatorname{Im}(g)=(\operatorname{Ker}(f) \cap \operatorname{Im}(g))$ is a direct summand of $M$. Since $\left.g\right|_{A^{\prime}}: A^{\prime} \longrightarrow \operatorname{Im}(g)$ is an isomorphism, we have $\left(\left.g\right|_{A^{\prime}}\right)^{-1}(\operatorname{Im}(g) \cap \operatorname{Ker}(f))$ is a direct summand of $M$. Hence by Lemma 1(b), $\operatorname{Ker}(f g)$ is a direct summand of $M$.

Recall that a module $M$ is called a C3-module if $A$ and $B$ are direct summands in $M$ with $A \cap B=0$, then $A \oplus B$ is a direct summand in $M$.

Following Ding et al. [16, Theorem 2.2(5)], a module $M$ is called a $C 4$-module if $A$ and $B$ are isomorphic direct summands in $M$ with $A \cap B=0$, then $A \oplus B$ is a direct summand in $M$. Clearly $C 3$-modules are $C 4$-modules. However, there are examples of $C 4$-modules which are not $C 3$.

The following theorem is an analogue of [15, Theorem 3.1].

Theorem 2. For a right $R$-module $M$, consider the following statements.

1. $M$ is C3-module.

2. For any two regular endomorphisms $f, g \in \operatorname{End}_{R}(M)$, if $\operatorname{Ker}(f g)$ is a direct summand of the module $M$, then the module $\operatorname{Im}(f g)$ is a direct summand of the module $M$.

3. For any two regular endomorphisms $f, g \in \operatorname{End}_{R}(M)$ satisfying the following:

(i) $\operatorname{Ker}(f g)$ is a direct summand of the module $M$,

(ii) $\operatorname{Ker}(f) \cong \operatorname{Im}(g)$,

then the module $\operatorname{Im}(f g)$ is a direct summand of the module $M$.

4. $M$ is a $C 4$-module.

5. For any two regular endomorphisms $f, g \in \operatorname{End}_{R}(M)$ satisfying the following:

(i) $\operatorname{Ker}(f g)$ is a direct summand of the module $M$,

(ii) $N \cong \operatorname{Im}(g)$ for any direct summand $N$ of $\operatorname{Ker}(f)$,

then the module $\operatorname{Im}(f g)$ is a direct summand of the module $M$.

Then $(1) \Leftrightarrow(2) \Rightarrow(3) \Rightarrow(4) \Rightarrow(5)$.

Proof. (1) $\Leftrightarrow(2)$ follows from [15, Theorem 3.1].

$(2) \Rightarrow(3)$ is clear.

$(3) \Rightarrow(4)$. Let $M=A \oplus A^{\prime}=B \oplus B^{\prime}$, where $A \cap B=0$ and $A \cong B$. Consider the natural projections $\pi_{1}: A \oplus A^{\prime} \longrightarrow A$ and $\pi_{2}: B \oplus B^{\prime} \longrightarrow B^{\prime}$. Then by Lemma 2(b), $\operatorname{Ker}\left(\pi_{2} \pi_{1}\right)=A^{\prime}$ is a direct summand of $M$. Therefore by assumption and Lemma 2(a), $\operatorname{Im}\left(\pi_{2} \pi_{1}\right)=(A+B) \cap B^{\prime}$ is a direct summand of $M$. Since $A+B=B \oplus(A+B) \cap B^{\prime}$, $A+B$ is a direct summand of $M$, as required.

$(4) \Rightarrow(5)$. Let

$$
M=\operatorname{Ker}(f) \oplus A=\operatorname{Im}(f) \oplus B=\operatorname{Ker}(g) \oplus A^{\prime}=\operatorname{Im}(g) \oplus B^{\prime} .
$$

By Lemma $1(\mathrm{~b}),\left(\left.g\right|_{A^{\prime}}\right)^{-1}(\operatorname{Im}(g) \cap K e r f)$ is a direct summand of $A^{\prime}$. Since $\left.g\right|_{A^{\prime}}: A^{\prime} \longrightarrow$ $\operatorname{Im}(g)$ is an isomorphism and $\operatorname{Im}(g)$ is a direct summand of the module $M$, we have that $\operatorname{Im}(g) \cap \operatorname{Ker}(f)$ is a direct summand of the module M. Therefore, $\operatorname{Ker}(f)=$ $N \oplus(\operatorname{Im}(g) \cap \operatorname{Ker}(f))$, for some $N \leq M$. Since $N \cap \operatorname{Im}(g)=0, N \cong \operatorname{Im}(g)$ and $M$ is a 
$C 4$-module, we have $N \oplus \operatorname{Im}(g)$ is a direct summand of $M$. Since $\operatorname{Ker}(f) \leq \operatorname{Im}(g) \oplus N$, we have that

$$
\operatorname{Im}(g) \oplus N=\operatorname{Ker}(f) \oplus(\operatorname{Im}(g)+N) \cap A=\operatorname{Ker}(f) \oplus(\operatorname{Im}(g)+\operatorname{Ker}(f)) \cap A .
$$

Therefore, $(\operatorname{Im}(g)+\operatorname{Ker}(f)) \cap A$ is a direct summand of $M$. Hence by Lemma 1(a), $\operatorname{Im}(f g)$ is a direct summand of $M$.

We can now prove the following result which has already appeared in $[17$, Proposition 5.7 and Corollary 2.9]. The proof has been outlined by us for the sake of completeness.

Proposition 3. For a right $R$-module $M$, the following conditions are equivalent.

1. $M$ is a D4-module and a SSP-module.

2. $M$ is a C3-module and a SIP-module.

3. $M$ is a C4-module and a SIP-module.

4. $M$ is a D3-module and a SSP-module.

5. $M$ is an SSP-module and a SIP-module.

Proof. (1) $\Longrightarrow(2)$. Let $M$ be a SSP-module. It is clear that $M$ is a $C 3$-module. To see that $M$ is a SIP-module, we shall use Proposition 2. Let $f, g \in \operatorname{End}_{R}(M)$ be two regular endomorphisms such that

$$
M=\operatorname{Ker}(f) \oplus A=\operatorname{Im}(f) \oplus B=\operatorname{Ker}(g) \oplus A^{\prime}=\operatorname{Im}(g) \oplus B^{\prime} .
$$

We need to show that $K e r(f g)$ is a direct summand of $M$. By Lemma 1(b), enough to show that $\operatorname{Im}(g) \cap K \operatorname{Ker}(f)$ is a direct sumand of $M$. To this end we shall follow the proof of [3, Proposition 1.4]. Let $\pi_{1}: \operatorname{Im}(g) \oplus B \longrightarrow \operatorname{Im}(g)$ and $\pi_{2}: \operatorname{Ker}(f) \oplus A \longrightarrow \operatorname{Ker}(f)$ be the natural projections. Define $\theta=\left.\left(\left(\pi_{1}-1\right) \circ \pi_{2}\right)\right|_{\operatorname{Im}(g)}: \operatorname{Im}(g) \longrightarrow B^{\prime}$. Then by $[2$, Proposition 1.4], $\operatorname{Im}(\theta)$ is a direct summand of $B^{\prime}$. Hence $M$ being a D4-module (use [7, Theorem 2.2]), we have $\operatorname{Ker}(\theta)=(\operatorname{Im}(g) \cap \operatorname{Ker}(f)) \oplus(\operatorname{Im}(g) \cap A)$ is a direct summand of $\operatorname{Im}(g)$. Thus $\operatorname{Im}(g) \cap \operatorname{Ker}(f)$ is a direct sumand of $M$, as desired.

$(2) \Longrightarrow(3)$ is clear.

$(3) \Longrightarrow(4)$. Let $M$ be a SIP-module. It is clear that $M$ is a $D 3$-module. To see that $M$ is a SSP-module, we shall use Proposition 1 . Let $f, g \in \operatorname{End}_{R}(M)$ be two regular endomorphisms such that

$$
M=\operatorname{Ker}(f) \oplus A=\operatorname{Im}(f) \oplus B=\operatorname{Ker}(g) \oplus A^{\prime}=\operatorname{Im}(g) \oplus B^{\prime} .
$$

We need to show that $\operatorname{Im}(f g)$ is a direct summand of $M$. By Lemma 1(a), enough to show that $\operatorname{Im}(g)+\operatorname{Ker}(f)$ is a direct sumand of $M$. To this end we shall follow the proof of [5, Theorem 8]. Let $\pi_{1}: \operatorname{Ker}(f) \oplus A \longrightarrow \operatorname{Ker}(f)$ and $\pi_{2}: \operatorname{Im}(g) \oplus B^{\prime} \longrightarrow B^{\prime}$ be the natural projections. Define $\phi=\left.\left(\pi_{2} \circ \pi_{1}\right)\right|_{\operatorname{Im}(g)}: \operatorname{Im}(g) \longrightarrow B^{\prime}$. Then by [3, Proposition 1.4], $\operatorname{Ker}(\phi)$ is a direct summand of $B^{\prime}$. Hence $M$ being a $C 4$-module (use [16, Theorem 2.2]), we have $\operatorname{Im}(\phi)=[\operatorname{Im}(g)+\operatorname{Ker}(f)] \cap[\operatorname{Im}(g)+A] \cap B^{\prime}$ is a direct summand of $\operatorname{Im}(g)$. So we can write $M=\operatorname{Im}(\phi) \oplus X$ for some $X \leq M$. Hence $B^{\prime}=\operatorname{Im}(\phi) \oplus\left(B^{\prime} \cap X\right)$. Then we have $M=[\operatorname{Im}(g)+\operatorname{Ker}(f)] \oplus\left[(\operatorname{Im}(g)+A) \cap\left(B^{\prime} \cap X\right)\right]$, as required.

$(4) \Longrightarrow(5)$ follows from Proposition 2 and Theorem 1 . 
$(5) \Longrightarrow(1)$ is clear.

The following result extends [15, Lemma 4.2(2)].

Proposition 4. Let $M$ be a dual Rickart module. If $M$ is a D4-module, then the product of any two regular elements in the ring $\operatorname{End}_{R}(M)$ is a regular element.

ProOF. It follows from the hypothesis and Proposition 3 that $M$ is a SSP-module and a SIP-module. Hence the result follows from [15, Theorem 2.7].

The following theorem was proved in [17].

Theorem 3 [17, Theorem 5.6]. Let $M=\oplus_{i \in I} M_{i}$ be a direct sum of submodules $M_{i}$. If $N=\oplus_{i \in I}\left(N \cap M_{i}\right)$ for every submodule $N$ of $M$, then $M$ is a D4-module if and only if for each $i \in I, M_{i}$ is a D4-module.

In [17], immediately after Theorem 3 the following question was asked.

Question (see [17, Question, p. 4494]). It is known that if $N=\oplus_{i \in I}\left(N \cap M_{i}\right)$ for every submodule $N$ of $M$, then $\operatorname{Hom}\left(M_{i}, M_{j}\right)=0$ for every $i \neq j$ in $I$, so it is natural to ask if [17, Theorem 5.6] (that is the theorem above) remains true if one assumes that $\operatorname{Hom}\left(M_{i}, M_{j}\right)=0$ for every $i \neq j$ in $I$.

In the next proposition we show that Question above has a positive answer.

Proposition 5. Let $M=\oplus_{i \in \mathbb{N}} M_{i}$ be a direct sum of submodules $M_{i}$ in which $\operatorname{Hom}\left(M_{i}, M_{j}\right)=0$ for every $i \neq j$. Then the following assertions hold:

(i) if $M$ is a D4-module, then for each $i \in I, M_{i}$ is a D4-module,

(ii) if each $M_{i}$ is a D4-module, then $M$ is a D4-module.

Proof. (i). Since a direct summand of a $D 4$-module is a $D 4$-module (see [7, Proposition 2.11]), for every $i \in \mathbb{N}, M_{i}$ is a $D 4$-module if $M$ is a $D 4$-module.

(ii). By hypothesis and [18, the paragraph before Corollary 16.5], we have

$$
\operatorname{End}_{R}(M) \cong\left(\begin{array}{ccccc}
\operatorname{End}_{R}\left(M_{1}\right) & 0 & 0 & . . & . . \\
0 & \operatorname{End}_{R}\left(M_{2}\right) & 0 & . . & . . \\
: & : & : & : & : \\
0 & 0 & . . & \operatorname{End}_{R}\left(M_{n}\right) & . . \\
: & : & : & : & \ddots
\end{array}\right)_{\mathbb{N} \times \mathbb{N}}
$$

Take two regular elements $f, g$ in $\operatorname{End}_{R}(M)$ such that $\operatorname{Im}(f g)$ is a direct summand of $M$ and $\operatorname{Ker}(f) \cong \operatorname{Im}(g)$. Then $f=\left(f_{i}\right)_{i \in \mathbb{N}}$ and $g=\left(g_{i}\right)_{i \in \mathbb{N}}$ for some regular elements $f_{i}$ and $g_{i}$ in $\operatorname{End}_{R}\left(M_{i}\right)$ such that $\operatorname{Im}\left(f_{i} g_{i}\right)$ is a direct summand of $M_{i}$ and $\left[X_{i}+\operatorname{Ker}\left(f_{i}\right)\right] \cong$ $\operatorname{Im}\left(g_{i}\right)$ for any direct summand $X_{i}$ of $M_{i}$ such that $X_{i} \cap \operatorname{Ker}\left(f_{i}\right)=0$ for all $i \in \mathbb{N}$. But then each $M_{i}$ is a $D 4$-module. Therefore by Theorem $1, \operatorname{Ker}\left(f_{i} g_{i}\right)$ is a direct summand of $M_{i}$ for all $i \in \mathbb{N}$. Hence $\operatorname{Ker}(f g)$ is a direct summand of $M$, as required.

Remark. Let $\left\{p_{i}\right\}_{i \in \mathbb{N}}$ be an infinite set of prime numbers and let $p$ be a prime different from any of them. Then we have the following examples of $D 4$-modules:

(i) $M=\mathbb{Z}_{p^{\infty}} \oplus\left(\oplus_{i \in \mathbb{N}} \mathbb{Z} / p_{i} \mathbb{Z}\right)$ as a $\mathbb{Z}$-module, where $\mathbb{Z}_{p^{\infty}}$ is the Prüfer $p$-group;

(ii) $M=\mathbb{Q} \oplus\left(\oplus_{i \in \mathbb{N}} \mathbb{Z} / p_{i} \mathbb{Z}\right)$ as a $\mathbb{Z}$-module. 
The author is grateful to the referee for a detailed list of suggestions and comments that helped improve the article significantly. Also, the author would like to thank Professor Yasser Ibrahim for some fruitful conversations and Professor M. B. Rege for his encouragement and help in presentation.

\section{References}

1. Kaplansky I. Infinite Abelian Groups. Ann Arbor, Univ. of Michigan Press (1969).

2. Garcia J. L. Properties of direct summands of modules. Comm. Algebra 17, 73-92 (1989). https://doi.org/10.1080/00927878908823714

3. Hausen J. Modules with the summand intersection property. Comm. Algebra 17, 135-148 (1989). https://doi.org/10.1080/00927878908823718

4. Wilson G. V. Modules with the direct summand intersection property. Comm. Algebra 14, 21-38 (1986).

5. Alkan M., Harmanci A. On summand sum and summand intersection property of modules. Turkish J. Math. 26, 131-147 (2002).

6. Mohamed S., Müller B. J. Continuous and Discrete Modules. In: London Math. Soc. Lecture Note Ser., vol. 147. Cambridge, Cambridge Univ. Press (1990).

7. Ding N., Ibrahim Y., Yousif M., Zhou Y. D4-modules. J. Algebra Appl. 16 (5), 1750166 (2017). https://doi.org/10.1142/S0219498817501663

8. Clark J., Lomp C., Vanaja N., Wisbauer R. Lifting modules: supplements and projectivity in module theory. Basel, Boston, Berlin, Birkhäuser, Verlag (2006). (2009).

9. Baba Y., Oshiro K. Classical Artinian Rings and Related Topics. World Scientific Publishing

10. Nguyen X. H., Yousif M., Zhou Y. Rings whose cyclics are D3-modules. J. Algebra Appl. 16 (8), 1750184 (2017). https://doi.org/10.1142/S0219498817501845

11. Ibrahim Y. Email communication (2020).

12. D'Este G., Keskin Tütüncü D., Tribak R. D3-modules versus D4-modules - Applications to quivers. Glasgow Math. Journal, 1-27 (2020). https://doi.org/10.1017/S0017089520000452

13. Lee G., Rizvi S. T., Roman C.S. Rickart modules. Comm. Algebra 38 (11) 4005-4027 (2010). https://doi.org/10.1080/00927872.2010.507232

14. Lee G., Rizvi S. T., Roman C. S. Dual Rickart modules. Comm. Algebra 39 (11), 4036-4058 (2011). https://doi.org/10.1080/00927872.2010.515639

15. Abyzov A. N., Tuganbaev A. A. Modules in which sums or intersections of two direct summands are direct summands. J. Math. Sciences 211 (3), 297-303 (2015). https://doi.org/10.1007/s10958-0152605-0

16. Ding N., Ibrahim Y., Yousif M., Zhou Y. C4-modules. Comm. Algebra 45 (4), 1727-1740 (2017). https://doi.org/10.1080/00927872.2016.1222412

17. Altun-Ozarslan M., Ibrahim Y., Özcan A. Ģ., Yousif M. C4- and D4-Modules via perspective direct summands. Comm. Algebra 46 (10), 4480-4497 (2018). https://doi.org/10.1080/00927872.2018. 1448838

18. Anderson F. W., Fuller K. R. Rings and Categories of Modules. In: Graduate Texts in Math., vol. 13. New York, Springer-Verlag (1974).

Received: September 13, 2020

Revised: March 14, 2021

Accepted: March 19, 2021

Author's information:

Soumitra Das - Assistant Professor; soumitrad330@gmail.com 


\section{K вопросу о D4-модулях}

\section{С. Дас}

Инженерно-технологический институт КПР, Коимбатур, 641407, Индия

Для цитирования: Das S. On a question concerning D4-modules // Вестник Санкт-Петербургского университета. Математика. Механика. Астрономия. 2021. Т. 8 (66). Вып. 3. C. 467-474. https://doi.org/10.21638/spbu01.2021.308

$R$-модуль $M$ называется $D 4$-модулем, если всякий раз, когда $M_{1}$ и $M_{2}$ являются прямыми слагаемыми $M$ с $M_{1}+M_{2}=M$ и $M_{1} \cong M_{2}$, то $M_{1} \backslash M_{2}$ является прямым слагаемым $M$. Пусть $M=\bigoplus_{i \in I} M_{i}$ - прямая сумма подмодулей $M_{i}$ с $\operatorname{Hom}\left(M_{i} ; M_{j}\right)=0$ для различных $i, j \in I$. Показано, что $M$ является $D 4$-модулем тогда и только тогда, когда для каждого $i \in I$ модуль $M_{i}$ является $D 4$-модулем. Это решает открытый вопрос о прямых суммах $D 4$-модулей. Наш подход не зависит от решения, полученного недавно Д’Эсте, Кескином Тютюнджу и Трибаком.

Ключевые слова: SIP-модули, D4-модули.

Статья поступила в редакцию 13 сентября 2020 г.; после доработки 14 марта 2021 г; рекомендована в печать 19 марта 2021 г.

Контактная информация:

Сумитра Дас-soumitrad330@gmail.com 